\title{
Remote Sensing Satellite Image Classification Using Neural Network
}

\author{
G.Karthik $^{1}$, M.Sangeetha ${ }^{2}$, B.Karthik ${ }^{3}$ \\ \{1 gkarthik7087@gmail.com, ${ }^{2}$ sang_gok@yahoo.com, ${ }^{3}$ karthikguru33@gmail.com $\}$ \\ ${ }^{1}$ Research Scholar, Department of Electronics \& Communication Engineering, Bharath Institute \\ of Higher Education and Research, Chennai, India \\ ${ }^{2}$ Professor, Department of Electronics \& Communication Engineering, Bharath Institute \\ of Higher Education and Research, Chennai, India \\ ${ }^{3}$ Associate Professor, epartment of Electronics \& Communication Engineering, Bharath Institute \\ of Higher Education and Research, Chennai, India
}

\begin{abstract}
Continuous monitoring of the geographical area of the Earth has become a necessity. Either to develop the infrastructure or to protect the resources of a region the right analysis of the geographical distribution has to be detected. Hence processing of satellite image of the region is the best solution. This paper proposes an approach for refining the detection and classification of various geographical regions like land, water, forest etc., in an area. Here the Convolutional Neural Network (CNN) classifier of deep learning technique is applied for processing the satellite image of the region under study using Matlab platform.
\end{abstract}

Keywords: Satellite, Classification, Convolution Neural Network, deep learning, Matlab.

\section{Introduction}

Global warming, Climatic changes, Industrial development, and Urbanization has made several changes to the landforms of the Earth. To know about the changes happened to a particular geographic region or to establish a makeover to a region terrain mapping is important. To study about the landforms like vegetation, water bodies, buildings present in a region an eagle eye view of that region is important. Remote sensing plays a major role for detection of physical features of the environment from a distant place like satellites. In satellite image processing the photograph of a particular region is captured in digital format and processed using machine vision to get the information required. It is a form of remote sensing where the pixel values are calculated for analysis. Multiple techniques and algorithms are developed to process the image, extract the facts required from the image and to classify them. Machine learning and deep learning are the trending techniques with multiple modules for categorizing an image with high range of accuracy. In remote sensing the common classification types are supervised classifiers, unsupervised classifiers and object based classifiers. The convolutional neural network classifier applied in this paper comes under both supervised and unsupervised category. 


\section{Literature Survey}

Due to the increasing need of terrain mapping many methods are developed and studied. Here, Vizireanu et al., 2017 [1] presents a study for comparing satellite images of three different resolution. Here the satellite image of Constanta city in Romania captured by three satellites namely Spot -5 Supermode, Landset 7 and Spot Multi-spectral are analysed by object-based classification using the software DefinienseCognition. The segmented images are classified based on the values obtained by defining the pixels of the images by fuzzy approximation. The results attained by comparing the classification of the images of three different resolution are tabulated. This output concludes that the accuracy of an image calculation directly depends on how well the resolution of the image is presented.

For the applications used for urban planning, a study of framework of the environment of an area is very important. But the data required for this purpose is not widely available due to its acquisition cost. Adrian Albert et al., 2017 [2] proposes a method which makes use of the large scale satellite images available from third party source. The use of convolutional neural network is examined in the environmental analysis of similarity and variability of 6 cities in Europe with 10 land classes. Here initially the land classes are predicted then this categorization is inserted with the factors obtained from convolution classifiers and converted into a continuous spectrum. Using this method any given image of the nearest area can be identified from the features extracted from the convolution method.

Nandhini, 2019 [3] describes about the various methods for classification of satellite images using cellular automata concept. Cell automation is the idea of arithmetic depiction of set of cells in matrix format. It is the recent technique used in simulation of images obtained using remote sensing. Here the processes involved in the execution of the basic classification types such as supervised, unsupervised and object orientated classifiers along with the techniques practiced are also outlined briefly. The aim of this paper was to infer the user about the type of classifier to be chosen to meet their requirement.

Eric Ariel et al., 2019 [4], proposes a novel idea for classifying agricultural system using random forest classifier algorithm based on the data set obtained from space view. Though many machine learning methods are used to define and analyse the hyperspectral data the accuracy is not fully guaranteed due to large bandwidth,. This may to lead to loss of data. In order to rectify this three new improved algorithms are developed and tested based on Moment Distance index. The execution of the algorithms are done on publicly available data set and obtained an improved accuracy range. This process is also considered to be time efficient.

Forest resources are widely destructed due to Forest fire. So the detection of this type of accident has to be monitored continuously for to take immediate action for rectification. Hence satellite images play a important role in this process. SreeSouthry et al., 2019 [5] proposed Supervised Multi-Model Image Classification Algorithm for immediate and accurate detection of forest fire. Here the before and after forest fire satellite images are obtained, preprocessed for noise removal and compared to get the difference values. Then it is segmented using Finite Image Clustering Segmentation algorithm and the features are extracted using Multi Spatial Feature Extraction method. The resulting dataset is classified using the SMICA technique. The implementation results show lower rate of error in detection of forest fire.

For image classification based on remote sensing, texture based classification features exhibits better results than vision based features. YinghuiQuan et al., 2020 [6], has formulated two types of modules for forest disaster management. In the first one, for feature extraction two types of widows are used instead of the traditional single window method. As the size of 
the widow determines the accuracy, one small enveloped by one large window is used. The extracted features are given to random Forest classifier for further processing. In the second type, from the features obtained only the important features are trained to be fed to classifier to decrease the computational time based on the decision tree classifier techniques.

In determination of the land boundaries the analysis of the historical maps is important. Here comes the satellite image processing techniques to aid this process. Anju Asokan et al., 2020, [7], has surveyed about various studies used for processing the satellite images to get the required data. A detail review of the types of techniques used and a clear roadmap of the tasks performed during the process is elaborately explained in this paper. Each system has its own unique way of proceedings and this determines the accuracy of the result. To avoid the complexity and challenges involved like low resolution of images, misapprehension of pixels etc., machine learning techniques are used. The paper concludes that further more researches have to be conducted to improve the robustness of the findings.

To analyse the environment of a geographical area many image processing methods have been developed. Here Anand et al., 2020 [8] proposes a method for detection of water level, crops, buildings in an area using the Support Vector Machine classifier algorithm in Matlab platform. The various steps involved in the detection process are explained here.

\section{Proposed System}

Classification of a surface type from an aerial image of a particular region involves three major steps like pre processing of image, segmenting and classifying based on the features. The following diagram represents the block diagram of our proposed system.

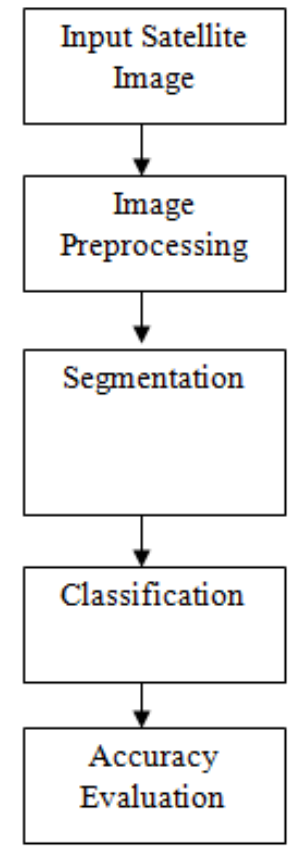

Fig 1: Proposed System Block Diagram 


\section{Preprocessing:}

To gain high accuracy results high resolution images have to be used in image processing. Considering this as a thumb rule, high quality satellite images of the area to be investigated can be obtained from any free data source like Google Map API. Cropping/ scaling of the original image is done to remove unwanted data and to reduce the complexity in handling large data. This cropped colour images undergoes colour normalization by converting the RGB bands to gray scale. In remote sensing the common disturbance in image acquisition is speckle noise which arises due to the environmental conditions. A non linear digital filter namely, the median filter is used here to remove the speckle noise. The median filter works by running through each signal entry and by interchanging it by the median of the nearby entry. It is widely used in image processing as it only removes the noise and does not alter the edges. The output of a median filter at any instant $t$ is the median of the input value corresponding to the instant next to $t$

$$
\mathrm{y}(\mathrm{t})=\operatorname{median}\left(\left(\mathrm{x}(\mathrm{t}-\mathrm{T} / 2), \mathrm{x}\left(\mathrm{t}-\mathrm{T}_{1}+1\right), \ldots, \mathrm{x}(\mathrm{t}), \ldots, \mathrm{x}(\mathrm{t}+\mathrm{T} / 2)\right) .\right.
$$

Where $\mathrm{x}$ - input, $\mathrm{y}$ - output, $\mathrm{t}$ - window size of the filter. The filtered image is enhanced to highlight the details so that the required data can be used efficiently during segmentation.

\section{Segmentation:}

Image segmentation is an important part of image processing. Here the images are partitioned into multiple regions based on the problem arising and this sub dividing process stops when the required objects are separated. Though many segmentation algorithms are present, the selection of the algorithm depends on the type and purpose of the image. In this paper thresholding technique is used for segmentation. This technique is classified into two types as global thresholding and local thresholding, where the former is sub classified as traditional, iterative and multistage thersholding. Here this segmentation splits the image into pixels based on the gray scale values to define the boundaries. It is expressed as,

$$
\begin{gathered}
\mathrm{T}=\mathrm{T}[\mathrm{x}, \mathrm{y}, \mathrm{p}(\mathrm{x}, \mathrm{y}), \mathrm{f}(\mathrm{x}, \mathrm{y}] \\
\mathrm{g}(\mathrm{x}, \mathrm{y})= \begin{cases}1 & \text { tf } f(x, y) \geq 1 \\
0 & \text { if } f(x, y) \leq 0\end{cases}
\end{gathered}
$$

Here $\mathrm{x}, \mathrm{y}$ are co -ordinate points, $\mathrm{T}$ is threshold values, $\mathrm{p}(\mathrm{x}, \mathrm{y})$ and $\mathrm{f}(\mathrm{x}, \mathrm{y})$ are the points of gray scale image pixels then $\mathrm{g}(\mathrm{x}, \mathrm{y})$ defines the threshold image.

\section{Classification}

Image classification is the process of segregating the pixels based on certain aspects required to fulfil the purpose of the experiment conducted. Classification of image mainly depends on the prior extraction of the required constraints. But in Convolutional Neural Network $(\mathrm{CNN})$, both extraction and classification falls under single frame. This makes CNN alluring as only the much needed features are extracted to perform categorization task. CNN comes under deep learning neural network and its functions represents the function of human brain carrying the signals through multi branched neurons for object recognition and classification. CNN is a multilayered function consisting of input layer, hidden layers and output layer. The hidden layers comprises of the following layers:

Covolutional layer: In this layer convolution operation is applied to the input data and the obtained data is passed to the next layer 


$$
\begin{aligned}
(f * g)(t) & \stackrel{\text { def }}{=} \int_{-\infty}^{\infty} f(\tau) g(t-\tau) d \tau \\
& =\int_{-\infty}^{\infty} f(t-\tau) g(\tau) d \tau .
\end{aligned}
$$

Convolution is the process of combing two functions ' $\mathrm{f}$ ' and ' $\mathrm{g}$ ' by integration. Here one function is used to modify the other one.

Relu and Pooling layers: The Relu is used to remove the non linearity and the pooling is used to merge the output of bunches of neuron into a single neuron of the preceding layer.

Fully connected layer: This connects every neuron in a layer to each neuron in the next layer.

The last fully connected layer gives the majority values reqiured for classes. In CNN hundreds of hidden layers are present which trains the features during operation. Based on the detected and trained features the classification id done.

\section{Results and Discussion}

Let us see in detail about the results of our proposed system. Figure 2 represents the input image that we are going to analyze. Figure 3 represents the preprocessing stage where our input image will be converted into grey scale image. Figure 4 represents the presence of speckle noise which has to be removed before segmentation as it will reduce the accuacy in predicting the segmented areas. Figure 5 represents the denoised image using adaptive filtering technique. Figure 6 represents the segmented image which is been obtained using threshold technique. Figure 7 represents the classified results obtained using Convolution Neural Network.

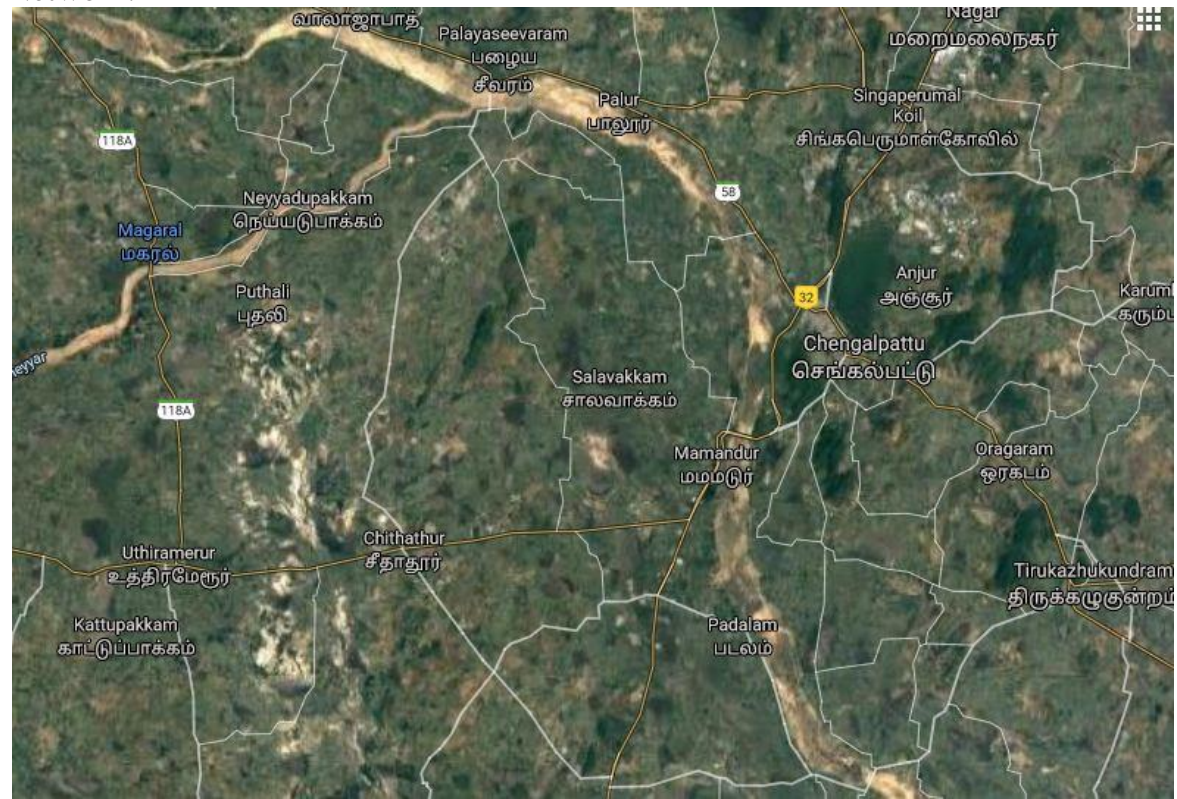

Fig 2: Input Satellite Image 


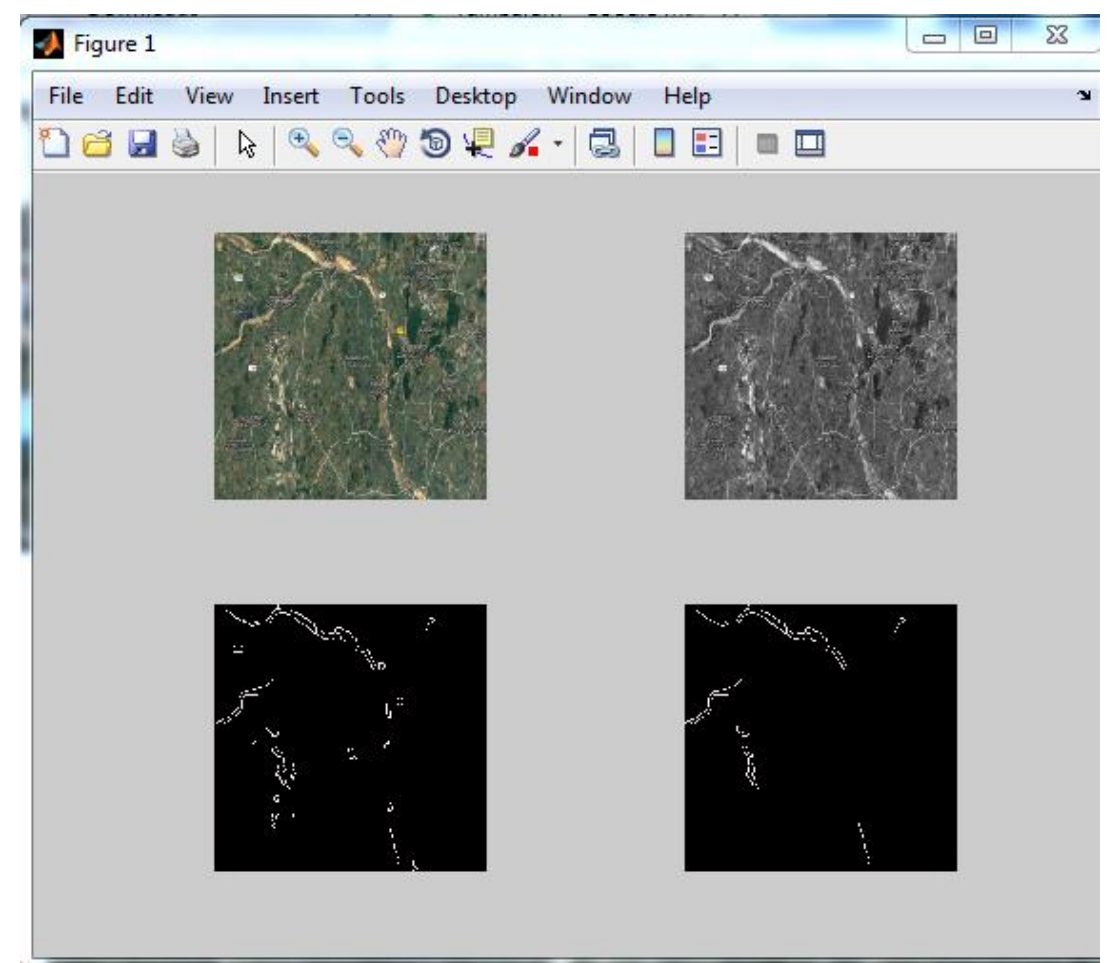

Fig 3: Preprocessing Stage

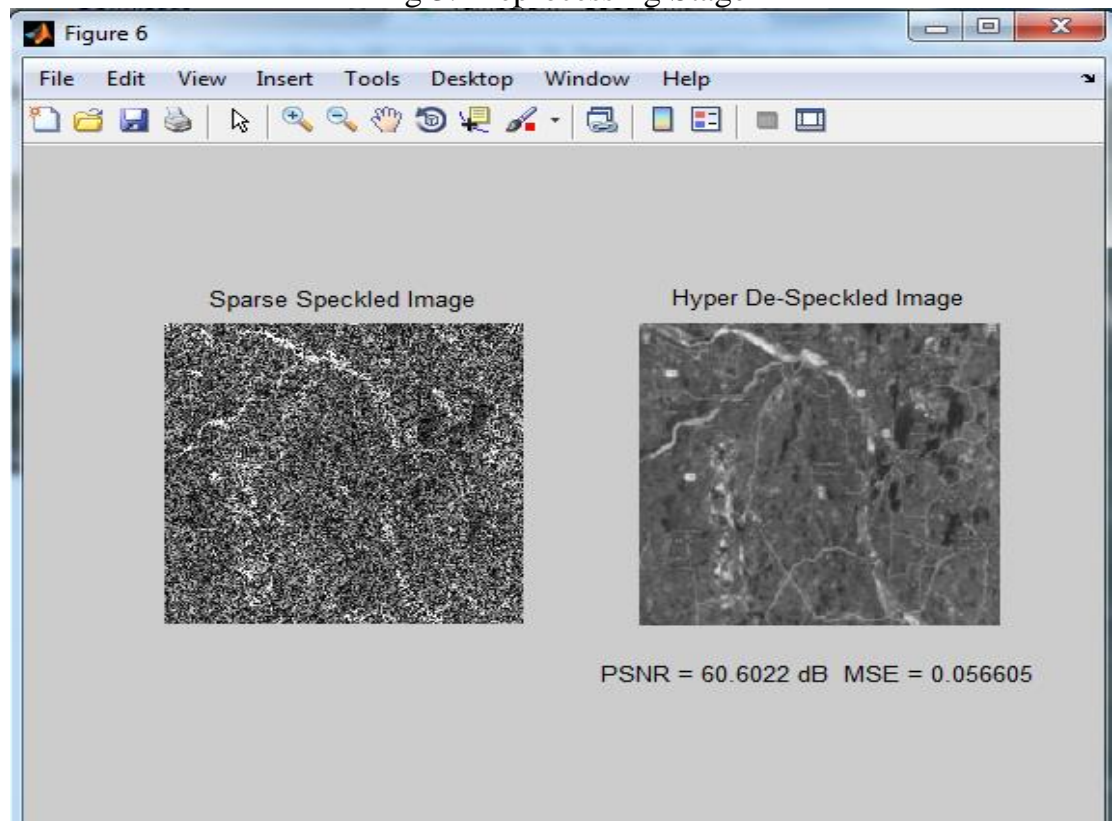

Fig 4: Presence of Noise 


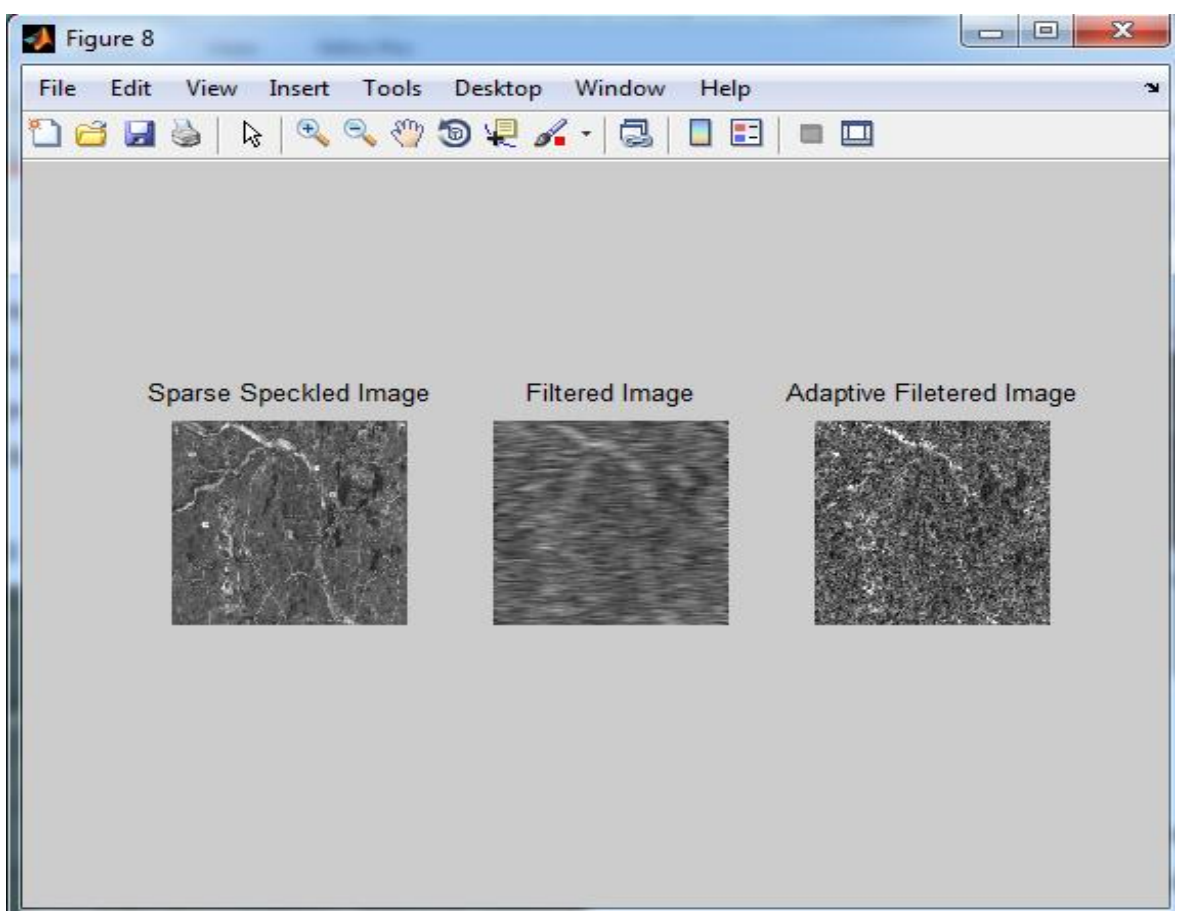

Fig 5: Denoised Image

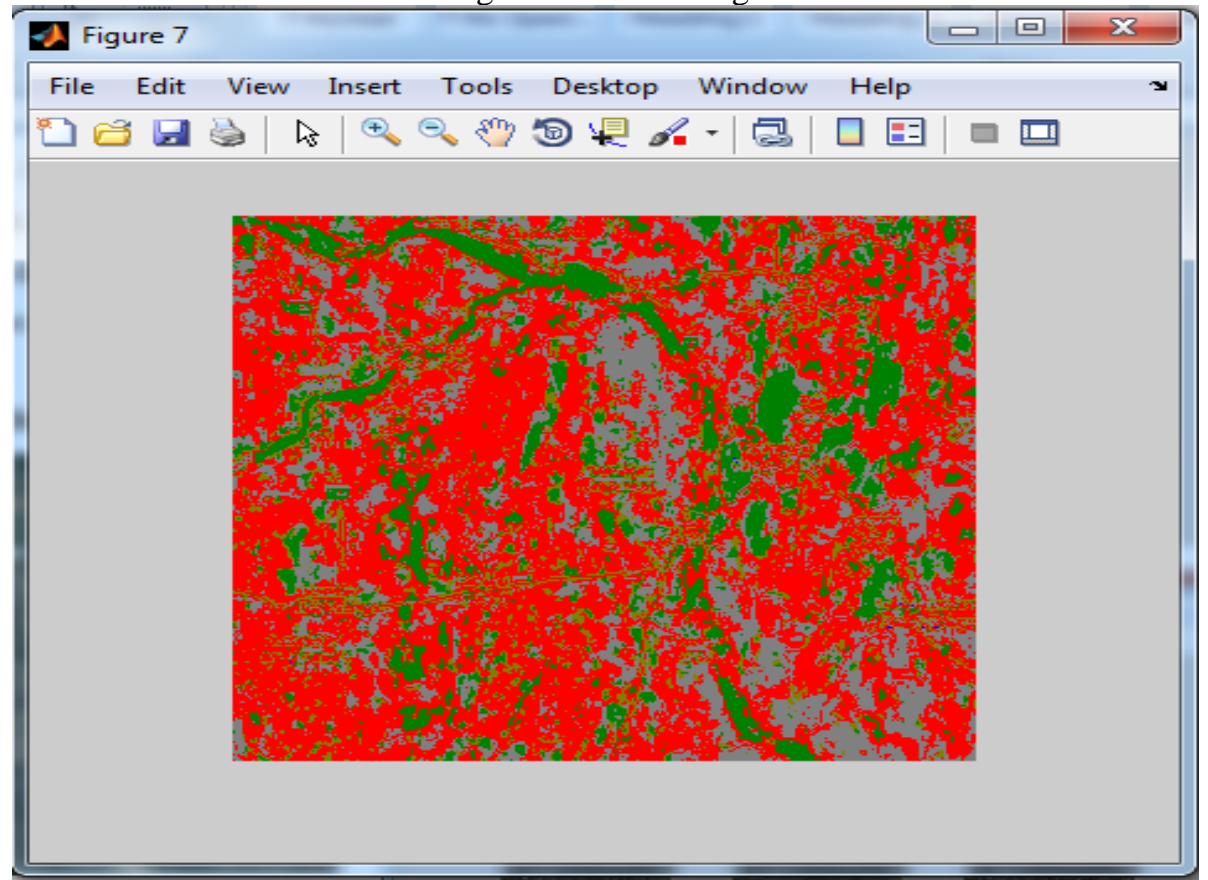

Fig 6: Segmented Image 


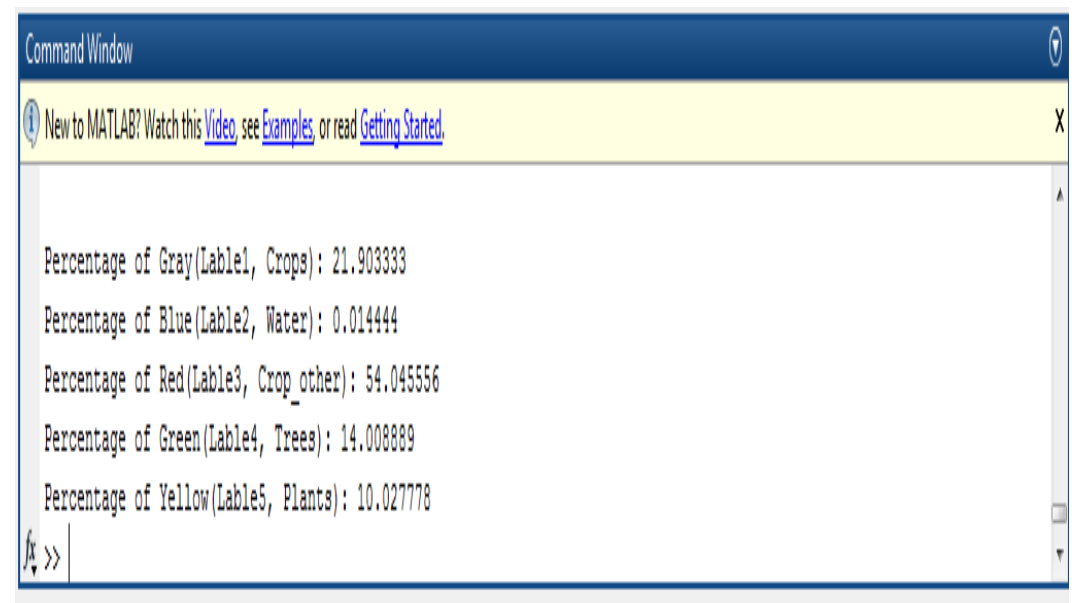

Fig 7: Output classification using $\mathrm{CNN}$

\section{Conclusion}

This paper has explored the operation of convolutional neural networks for classification of satellite image for analysing the environmental changes. It proves our proposed system is very efficient in noise removal and segmentation is also done in better manner. The output of classification is too better than any other classification methods.

\section{References}

[1] Vizireanu. I , Mateescu.R, Golumbeanu.M, Benciu.S, Slave.C (2017), "Environmental Applications of Object-Based Classification Techniques in Time Series Analysis of Different Resolution Satellite Images”, Journal of Environmental Protection and Ecology 18, No 1, pp.181190

[2] Adrian Albert, Jasleen Kaur, Marta C. Gonz'alez (2017), "Using Convolutional Networks and Satellite Imagery to Identify Patterns in Urban Environments at a Large Scale", Proceedings of the 23rd ACM SIGKDD International Conference on Knowledge Discovery and Data Mining, DOI:10.1145/3097983.3098070

[3] Nandhini (2019), "Classification of satellite images using cellular automata ", International Journal of Advance Research, Ideas and Innovations in Technology, Vol. 5, Issue 3 ISSN: 2454-132X

[4] Eric Ariel L. Salas, Sakthi Kumaran Subburayalu (2019), "Modified shape index for object-based random forest image classification of agricultural systems using airborne hyperspectral datasets", PLOS.ONE | https://doi.org/10.1371/journal.pone.0213356

[5] SreeSouthry, S. Vinayagapriya, A. Ayub Khan, P. Srinivasan (2020), "A Highly Accurate and Fast Identification of Forest Fire Based on Supervised Multi Model Image Classification Algorithm (SMICA)", Journal of Critical Reviews, Vol 7, Issue 6, ISSN- 2394-5125

[6] Karthik, B., Kumar, T.K., Dorairangaswamy, M.A., Logashanmugam, E., Removal of high density salt and pepper noise through modified cascaded filter, Middle - East Journal of Scientific Research, 2014, 20(10), pp. 1222-1228

[7] YinghuiQuan, Xian Zhong, Wei Feng, Gabriel Dauphin , Lianru Gao, Mengdao Xing (2020), “A Novel Feature Extension Method for the Forest Disaster Monitoring Using Multispectral Data", Remote Sensing, doi:10.3390/rs12142261 
[8] Anju Asokan, J. Anitha, Monica Ciobanu, Andrei Gabor, AntoanelaNaaji, D. Jude Hemanth, (2020), "Image Processing Techniques for Analysis of Satellite Images for Historical Maps Classification-An Overview", Applied Sciences. 2020, 10, 4207; doi:10.3390/app10124207

[9] Karthik, B., Krishna Kumar, T., Vijayaragavan, S.P., Sriram, M., Removal of high density salt and pepper noise in color image through modified cascaded filter, Journal of Ambient Intelligence and Humanized Computing, 2020

[10] Anand.R, Dhinakaran K, Dharani.G, Gayathri.P, Laila.Y, (2020), "Satellite Image Classification and Segmentation For Environmental Analyses And Safety", International Journal of Advance Science and Technology, Vol. 29, No. 10S, (2020), pp. 7318-7322, ISSN: 2005-4238

[11] Mohammed Abbas Kadhim and Mohammed Hamzah Abed, (2020), "Convolutional Neural Network for Satellite Image Classification”, https://doi.org/10.1007/978-3-030-14132-5_13

[12] Goutham Reddy, L. Rama Parvathy, (2020), "Satellite Image Classification for Environmental Analysis using Image Processing", International Journal of Innovative Technology and Exploring Engineering, Vol.-9 Issue-3, ISSN: 2278-3075

[13] Karthik, B., Kiran Kumar, T.V.U., Noise removal using mixtures of projected gaussian scale mixtures, World Applied Sciences Journal, 2014, 29(8), pp. 1039-1045 